\title{
OPTIMAL ASYMPTOTIC ESTIMATES FOR THE VOLUME OF INTERNAL INHOMOGENEITIES IN TERMS OF MULTIPLE BOUNDARY MEASUREMENTS
}

\author{
Yves CapdeboscQ $^{1}$ and Michael S. Vogelius ${ }^{1}$
}

\begin{abstract}
We recently derived a very general representation formula for the boundary voltage perturbations caused by internal conductivity inhomogeneities of low volume fraction ( $c f$. Capdeboscq and Vogelius (2003)). In this paper we show how this representation formula may be used to obtain very accurate estimates for the size of the inhomogeneities in terms of multiple boundary measurements. As demonstrated by our computational experiments, these estimates are significantly better than previously known (single measurement) estimates, even for moderate volume fractions.
\end{abstract}

Mathematics Subject Classification. 35J20, 35B27, 35R30.

Received: September 6, 2002. Revised: November 6, 2002.

\section{INTRODUCTION AND MAIN RESULTS}

The problem of identifying internal inhomogeneities based on a few boundary measurements has received quite a bit of attention ( $c f .[3,4,6,7,9-12]$ and [15]). One particular line of inquiry has been concerned with the estimation of size based on a single boundary integral ( $c f .[1,2]$ and [13]). The main result in [1] provides optimal upper and lower bounds on the volume of the set of internal inhomogeneities in terms of a single integral (measurement) which represents the difference in "total work" when compared to the homogeneous situation. It seems quite natural to ask if (and how) one may improve such estimates for the volume of the inhomogeneities if one is permitted to make multiple measurements. The goal in this paper is to provide an answer to this question in the case of $m$ measurements (boundary integrals) for a domain $\Omega \subset \mathbb{R}^{m}$. The simple, improved estimates we derive are shown to be rigorous, improved bounds in the asymptotic limit when the inhomogeneities are of small relative volume, and furthermore, computational evidence demonstrates that they are significantly closer to the actual volume than the one-measurement estimates, even for moderate volume fractions. It remains an interesting open problem to design improved, multiple measurement estimates using a number of measurements different from the dimension, $m$. It should also be interesting to investigate if effective multiple measurement estimates may be designed in such a way, that they truly bound the volume without any (asymptotic) size restrictions.

Consider a conducting object which occupies a bounded, smooth domain $\Omega \subset \mathbb{R}^{m}$, and which conducts direct currents. Let $\gamma_{0}(\cdot)$ denote the smooth background conductivity, that is, the conductivity in the absence of any

Keywords and phrases. Conductivity inhomogeneities, volume estimates, low volume fraction.

1 Department of Mathematics, Rutgers University, New Brunswick, NJ 08903, USA. e-mail: vogelius@hilbert.rutgers.edu

(C) EDP Sciences, SMAI 2003 
inhomogeneities. We suppose that

$$
0<c_{0} \leq \gamma_{0}(x) \leq C_{0}<\infty, \quad x \in \Omega
$$

for some fixed constants $c_{0}$ and $C_{0}$. The function $\psi$ denotes the imposed boundary current. It suffices that $\psi \in H^{-1 / 2}(\partial \Omega)$, with $\int_{\partial \Omega} \psi \mathrm{d} \sigma=0$. The background voltage potential, $U$, is the solution to the boundary value problem

$$
\begin{aligned}
& \nabla \cdot\left(\gamma_{0}(x) \nabla U\right)=0 \text { in } \Omega, \\
& \gamma_{0}(x) \frac{\partial U}{\partial n}=\psi \text { on } \partial \Omega \text {. }
\end{aligned}
$$

Here $n$ denotes the unit outward normal to the domain $\Omega$.

Let $\omega_{\epsilon}$ denote a set of "inhomogeneities" inside $\Omega$. The geometric assumptions about the set of "inhomogeneities" are very simple: we suppose $\omega_{\epsilon}$ is measurable, and separated away from the boundary, in the sense that

$$
\operatorname{dist}\left(\omega_{\epsilon}, \partial \Omega\right)>d_{0}>0 .
$$

Most importantly, we suppose that $0<\left|\omega_{\epsilon}\right|$ is small, where $\left|\omega_{\epsilon}\right|$ denotes the Lebesgue measure of $\omega_{\epsilon}$. It is in order to emphasize this smallness assumption that we have decided to use the subscript $\epsilon$. Let $\hat{\gamma}_{\epsilon}$ denote the conductivity profile in the presence of the inhomogeneities. The function $\hat{\gamma}_{\epsilon}$ is equal to $\gamma_{0}$, except on the set of inhomogeneities; on the set of inhomogeneities we suppose that $\hat{\gamma}_{\epsilon}$ equals the restriction of some other smooth function, $\gamma_{1}$, with

$$
0<c_{1} \leq \gamma_{1}(x) \leq C_{1}<\infty, \quad x \in \Omega .
$$

In other words

$$
\hat{\gamma}_{\epsilon}(x)= \begin{cases}\gamma_{0}(x), & x \in \Omega \backslash \omega_{\epsilon} \\ \gamma_{1}(x), & x \in \omega_{\epsilon} .\end{cases}
$$

The voltage potential in the presence of the inhomogeneities is denoted $u_{\epsilon}(x)$. It is the solution to

$$
\begin{aligned}
& \nabla \cdot\left(\hat{\gamma}_{\epsilon}(x) \nabla u_{\epsilon}\right)=0 \text { in } \Omega \\
& \hat{\gamma}_{\epsilon}(x) \frac{\partial u_{\epsilon}}{\partial n}=\psi \text { on } \partial \Omega \text {. }
\end{aligned}
$$

We normalize both $\mathrm{U}$ and $u_{\epsilon}$ by requiring that

$$
\int_{\partial \Omega} U \mathrm{~d} \sigma=0, \quad \text { and } \quad \int_{\partial \Omega} u_{\epsilon} \mathrm{d} \sigma=0 .
$$

For $y \in \Omega$ let $N(\cdot, y)$ be the the solution to the boundary value problem

$$
\begin{aligned}
& \nabla_{x} \cdot\left(\gamma_{0}(x) \nabla_{x} N(x, y)\right)=\delta_{y} \text { in } \Omega, \\
& \gamma_{0}(x) \frac{\partial N}{\partial n_{x}}=\frac{1}{|\partial \Omega|} \text { on } \partial \Omega .
\end{aligned}
$$


The function $N(x, y)$ may be extended by continuity to $y \in \bar{\Omega}$. For $y \in \partial \Omega$ the function $N(\cdot, y)$ may also be interpreted as the solution to the boundary value problem

$$
\begin{aligned}
& \nabla_{x} \cdot\left(\gamma_{0}(x) \nabla_{x} N(x, y)\right)=0 \text { in } \Omega, \\
& \gamma_{0}(x) \frac{\partial N}{\partial n_{x}}=-\delta_{y}+\frac{1}{|\partial \Omega|} \text { on } \partial \Omega .
\end{aligned}
$$

In the recent paper [8] we derived a representation formula for (all possible limits of) $\left(u_{\epsilon}-U\right) \mid \partial \Omega$ as $\left|\omega_{\epsilon}\right| \rightarrow 0$.

Theorem 1. Let $\omega_{\epsilon_{n}}$ be a sequence of measurable subsets, with $\left|\omega_{\epsilon_{n}}\right| \rightarrow 0$, for which (2) holds. Given any $\psi \in H^{-1 / 2}(\partial \Omega)$, with $\int_{\partial \Omega} \psi \mathrm{d} \sigma=0$, let $U$ and $u_{\epsilon_{n}}$ denote the solutions to (1) and (3), respectively. There exists a subsequence, also denoted $\omega_{\epsilon_{n}}$, a regular probability measure, $\mu$, and a matrix valued function $M \in L^{2}(\Omega, \mathrm{d} \mu)$ such that

$$
\left(u_{\epsilon_{n}}-U\right)(y)=\left|\omega_{\epsilon_{n}}\right| \int_{\Omega}\left(\gamma_{1}-\gamma_{0}\right)(x) M_{i j}(x) \frac{\partial U}{\partial x_{i}} \frac{\partial N}{\partial x_{j}}(x, y) \mathrm{d} \mu(x)+o\left(\left|\omega_{\epsilon_{n}}\right|\right) \quad y \in \partial \Omega .
$$

The values of the function $M(\cdot)$ are symmetric, positive definite matrices $\mu$ almost everywhere in the set $\left\{x: \gamma_{0}(x) \neq \gamma_{1}(x)\right\}$. The subsequence $\omega_{\epsilon_{n}}$, the probability measure $\mu$, and the function $M \in L^{2}(\Omega, \mathrm{d} \mu)$ are independent of the boundary flux $\psi$. The term $o\left(\left|\omega_{\epsilon_{n}}\right|\right)$ is such that $\left\|o\left(\left|\omega_{\epsilon_{n}}\right|\right)\right\|_{L^{\infty}(\partial \Omega)} /\left|\omega_{\epsilon_{n}}\right|$ converges to 0 for any fixed $\psi \in H^{-1 / 2}$, and uniformly on $\left\{\psi: \int_{\partial \Omega} \psi \mathrm{d} \sigma=0, \quad\|\psi\|_{L^{2}(\partial \Omega)} \leq 1\right\}$.

It is clear that there is not in general an asymptotic representation formula for the entire original sequence $\omega_{\epsilon_{n}}$; different subsequences may be associated with different measures $\mu$ and different polarization tensors $M(\cdot)$. The measures $\mu$ have compact support (contained in $\left\{x \in \Omega: \operatorname{dist}(x, \partial \Omega) \geq d_{0}\right\}$ ), and it is possible to establish bounds for the tensor $M$ ( $\mu$ almost everywhere in the set $\left.\left\{x: \gamma_{0}(x) \neq \gamma_{1}(x)\right\}\right)$. Indeed in $[8]$ we proved that

$$
\min \left\{1, \frac{\gamma_{0}}{\gamma_{1}}\right\}|\xi|^{2} \leq M_{i j} \xi_{i} \xi_{j} \leq \max \left\{1, \frac{\gamma_{0}}{\gamma_{1}}\right\}|\xi|^{2} .
$$

Let $a\left(\frac{\gamma_{0}}{\gamma_{1}}\right)$ and $h\left(\frac{\gamma_{0}}{\gamma_{1}}\right)$ denote the averages given by

$$
a\left(\frac{\gamma_{0}}{\gamma_{1}}\right)=\frac{1}{m}\left(m-1+\frac{\gamma_{0}}{\gamma_{1}}\right), \quad h\left(\frac{\gamma_{0}}{\gamma_{1}}\right)=m\left(m-1+\frac{\gamma_{1}}{\gamma_{0}}\right)^{-1} .
$$

This is the arithmetic average and the harmonic average of $m-1$ copies of 1 and one copy of $\frac{\gamma_{0}}{\gamma_{1}}$, respectively. In the last section of this paper we shall derive an improved bound for the trace of $M$, namely

$$
m h\left(\frac{\gamma_{0}}{\gamma_{1}}\right) \leq \operatorname{trace}(M) \leq m a\left(\frac{\gamma_{0}}{\gamma_{1}}\right) .
$$

We note that

$$
\int_{\partial \Omega} \frac{\partial N}{\partial x_{j}}(x, y) \psi(y) \mathrm{d} \sigma_{y}=-\frac{\partial U}{\partial x_{j}}(x)
$$

Multiplication of (4) by $\psi$ and integration over $\partial \Omega$ thus yields the following formula for the "difference in total work"

$$
\int_{\partial \Omega}\left(u_{\epsilon_{n}}-U\right) \psi \mathrm{d} \sigma=-\left|\omega_{\epsilon_{n}}\right| \int_{\Omega}\left(\gamma_{1}-\gamma_{0}\right) M_{i j} \frac{\partial U}{\partial x_{i}} \frac{\partial U}{\partial x_{j}} \mathrm{~d} \mu+o\left(\left|\omega_{\epsilon_{n}}\right|\right) .
$$


From now on we shall assume that the known conductivities $\gamma_{0}$ and $\gamma_{1}$ are constants, with $\gamma_{0} \neq \gamma_{1}$. If $\psi$ is not identically zero, then introduction of the bounds (5) into (7) leads to

$$
\begin{aligned}
\min \left\{1, \frac{\gamma_{1}}{\gamma_{0}}\right\} \frac{1}{\left|\gamma_{1}-\gamma_{0}\right| \max |\nabla U|^{2}}\left|\int_{\partial \Omega}\left(u_{\epsilon}-U\right) \psi \mathrm{d} \sigma\right| & \leq\left|\omega_{\epsilon}\right|(1+o(1)) \\
& \leq \max \left\{1, \frac{\gamma_{1}}{\gamma_{0}}\right\} \frac{1}{\left|\gamma_{1}-\gamma_{0}\right| \min |\nabla U|^{2}}\left|\int_{\partial \Omega}\left(u_{\epsilon}-U\right) \psi \mathrm{d} \sigma\right|
\end{aligned}
$$

Consider in particular the boundary currents $\psi^{(i)}=\gamma_{0} n_{i}, 1 \leq i \leq m$, with corresponding potentials $u_{\epsilon}^{(i)}$ and $U^{(i)}=x_{i}+$ const. Let $D^{(i)}$ denote the boundary data corresponding to these currents

$$
D^{(i)}=\int_{\partial \Omega}\left(u_{\epsilon}^{(i)}-U^{(i)}\right) \psi^{(i)} \mathrm{d} \sigma=\gamma_{0} \int_{\partial \Omega}\left(u_{\epsilon}^{(i)}-x_{i}\right) n_{i} \mathrm{~d} \sigma, \quad 1 \leq i \leq m .
$$

Here repeated indices do not indicate summation. The preceeding bounds for the volume of the inhomogeneities now translate into

Asymptotic estimates for $\left|\omega_{\epsilon}\right|$ in terms of a single measurement, $D^{(i)}$ :

$$
\min \left\{1, \frac{\gamma_{1}}{\gamma_{0}}\right\} \frac{\left|D^{(i)}\right|}{\left|\gamma_{1}-\gamma_{0}\right|} \leq\left|\omega_{\epsilon}\right|(1+o(1)) \leq \max \left\{1, \frac{\gamma_{1}}{\gamma_{0}}\right\} \frac{\left|D^{(i)}\right|}{\left|\gamma_{1}-\gamma_{0}\right|}
$$

In order to obtain size estimates based on $m$ simultaneous measurements, we simply form the sum of (7) with $\psi=\gamma_{0} n_{i}$, for $i=1 \ldots m$,

$$
\sum_{i=1}^{m} D^{(i)}=\sum_{i=1}^{m} \gamma_{0} \int_{\partial \Omega}\left(u_{\epsilon_{n}}^{(i)}-x_{i}\right) n_{i} \mathrm{~d} \sigma=-\left|\omega_{\epsilon_{n}}\right| \int_{\Omega}\left(\gamma_{1}-\gamma_{0}\right) \operatorname{trace}(M) \mathrm{d} \mu+o\left(\left|\omega_{\epsilon_{n}}\right|\right) .
$$

Introduction of the bounds (6) (or more precisely the integral bounds $m h\left(\frac{\gamma_{0}}{\gamma_{1}}\right) \leq \int \operatorname{trace}(M) \mathrm{d} \mu \leq m a\left(\frac{\gamma_{0}}{\gamma_{1}}\right)$ ) now leads to the following

Asymptotic estimates for $\left|\omega_{\epsilon}\right|$ in terms of a $m$ simultaneous measurements, $D^{(i)}, 1 \leq i \leq m$ :

$$
\frac{1}{a\left(\frac{\gamma_{0}}{\gamma_{1}}\right)} \frac{\left|\sum_{i=1}^{m} D^{(i)}\right|}{m\left|\gamma_{1}-\gamma_{0}\right|} \leq\left|\omega_{\epsilon}\right|(1+o(1)) \leq \frac{1}{h\left(\frac{\gamma_{0}}{\gamma_{1}}\right)} \frac{\left|\sum_{i=1}^{m} D^{(i)}\right|}{m\left|\gamma_{1}-\gamma_{0}\right|}
$$

The main conclusion of this paper is that the estimates (9), even for moderate size volume, represent a significant improvement over (8). We demonstrate this by means of computational experiments in the next section. The only point in the derivation of (9) that is still missing is the verification of the bounds (6). This verification is found in Section 3 of this paper. To minimize the amount of technicalities we only present the verification in the case when $\gamma_{0} \neq \gamma_{1}$, and $M$ are all constant (the latter naturally only on the support of $\mu$ ). As pointed out in Remark 2 of Section 3, what we rigorously establish for non-constant $M$ (but constant $\gamma_{0}$ and $\gamma_{1}$ ) is that

$$
m h\left(\frac{\gamma_{0}}{\gamma_{1}}\right) \leq \int_{\Omega} \operatorname{trace}(M) \mathrm{d} \mu \leq m a\left(\frac{\gamma_{0}}{\gamma_{1}}\right)
$$

which incidentally is exactly what we needed in our derivation of the $m$-measurement estimates (9). Our approach is a variational one, the likes of which are often associated with the names of Hashin and Shtrikman.

The one-measurement estimates (8) are indeed true upper and lower bounds irrespective of the size of the volume (see [1] and also [13]). This is not the case for the $m$-measurement estimates (9), as we shall see in the next section. 

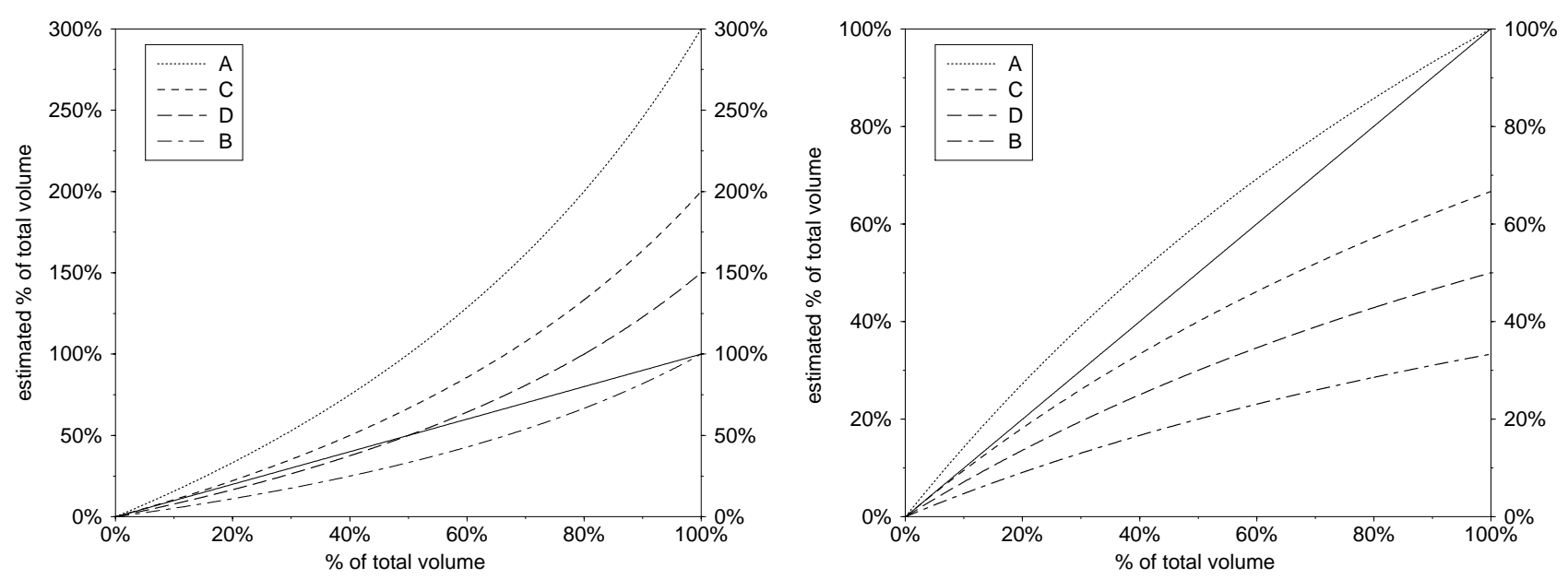

FiguRE 1. The four volume estimates in the case of a single concentric circular inhomogeneity. The background conductivity, $\gamma_{0}$, is 1 . For the left graph $\gamma_{1}$, the conductivity of the inhomogeneity, is $1 / 3$, for the right graph $\gamma_{1}$ is 3 . The curves A and B represent the one-measurement estimates (8); the curves $\mathrm{C}$ and $\mathrm{D}$ the two-measurement estimates (9).

The estimates provided here concern inhomogeneities of finite, non-zero conductivity. It should be interesting to find similar estimates for inhomogeneities of extreme conductivity. Since the zero volume limit of the voltage potentials in that case is not always equal to $U$, such estimates most likely will require extra geometric assumptions about $\omega_{\epsilon}$ - maybe in the spirit of those imposed in [2], in order to extend the one-measurement bounds. The estimates presented here immediately carry over to the case of the Helmholtz equation - and with some modifications very likely also to the full time-harmonic Maxwell equations.

\section{Computational experiments}

The main goal of this section is to provide computational evidence of the increased accuracy of the estimates based on multiple measurements. However, before we proceed with this task we want to illustrate a point we made earlier, namely that (9) are only true bounds in the asymptotic sense. To see this let $\Omega \subset \mathbb{R}^{2}$ be the unit disk and consider a single inhomogeneity in the form of a concentric disk of radius $0<r<1$. In Figure 1 we display the actual volume of the inhomogeneity versus each of the four bounds in (8) and (9) (expressed as a percentage of the total volume of $\Omega$ ). For easy comparison we also plot the line of slope one, which represents the best possible estimate for $\left|\omega_{\epsilon}\right|$, namely its exact value. We take $\gamma_{0}=1$. The left graph in Figure 1 corresponds to $\gamma_{1}=1 / 3$, the right graph corresponds to $\gamma_{1}=3$. The curves $\mathrm{A}$ and $\mathrm{B}$ represent the upper and lower onemeasurement estimates from (8). The curves $\mathrm{C}$ and $\mathrm{D}$ represent the upper and lower two-measurement estimates from (9). As pointed out earlier the curves A and B are true bounds for any size inhomogeneity. Concerning the upper two-measurement estimate (curve C) the right graph clearly shows that this may indeed be strictly below the exact volume for any positive value. For the present family of concentric circular inclusions the lower two-measurement estimate (curve D) is, for sufficiently small volume, a true lower bound. For relatively large volume this lower estimate may, however, strictly exceed the exact value, as evidenced in the graph to the left. We can construct examples where the lower two-measurement estimate fails to be a strict lower bound before the upper estimate fails to be a strict upper bound, by replacing the dilated circles in the present example with dilated ellipses of a sufficiently high aspect ratio.

We note that even if they are not true bounds the two-measurement estimates are still closer to the true volume, than the one-measurement estimates, for relative volumes fractions up to $20 \%$. 


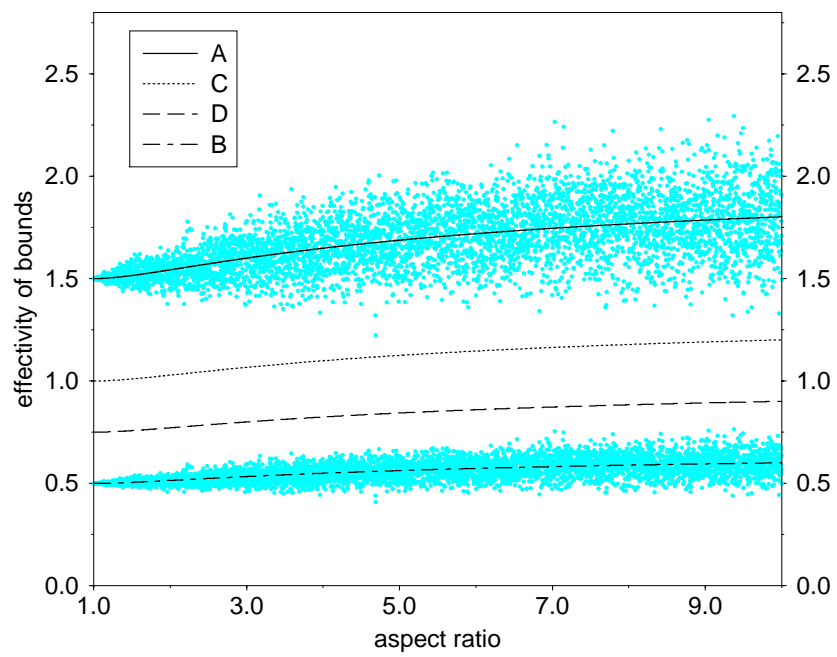

FigURE 2. The asymptotic effectivities of the four volume estimates as a function of the aspect ratio, $\rho$. The clusters around the curves $\mathrm{A}$ and $\mathrm{B}$ correpond to the one-measurement estimates (8). The curves A and B themselves corespond to the "average" one-measurement estimates. The curves C and D correspond to the two-measurement estimates (9).

The $m$-measurement estimates were designed to be highly accurate in the limit as the volume fraction of inhomogenities approaches 0 . Our next experiment is intended to quantify this statement. We consider again the two dimensional case $(m=2)$ and we take sets of inhomogeneities in the form of (finite) unions of infinitesimally small ellipses. We suppose all the ellipses (in one set of inhomogeneities) are identical and of aspect ratio $\rho$ (by the aspect ratio of an ellipse we mean the ratio between its major and minor axis). The orientations and the locations are arbitrary, subject only to the condition that the ellipses stay well separated and away from the boundary. Due to these assumptions we may use the first term in the representation formula (7) to approximate the boundary data $D^{(i)}$. For an explicit formula for $M$ in the case of ellipses, see [7]. If we suppose there are $K$ ellipses and that the major axis of $j$ th ellipse is rotated by an angle of $\theta_{j}$ counterclockwise from the $x_{1}$ axis, then it is easy to calculate that $D^{(i)}, i=1,2$, are well approximated by

$$
\tilde{D}^{(1)}=-\frac{\left|\omega_{\epsilon}\right|}{K} \sum_{j=1}^{K}\left(\gamma_{1}-\gamma_{0}\right) \gamma_{0}(\rho+1)\left[\frac{\cos ^{2} \theta_{j}}{\gamma_{0} \rho+\gamma_{1}}+\frac{\sin ^{2} \theta_{j}}{\gamma_{0}+\gamma_{1} \rho}\right]
$$

and

$$
\tilde{D}^{(2)}=-\frac{\left|\omega_{\epsilon}\right|}{K} \sum_{j=1}^{K}\left(\gamma_{1}-\gamma_{0}\right) \gamma_{0}(\rho+1)\left[\frac{\sin ^{2} \theta_{j}}{\gamma_{0} \rho+\gamma_{1}}+\frac{\cos ^{2} \theta_{j}}{\gamma_{0}+\gamma_{1} \rho}\right]
$$

Let $U_{1}, L_{1}$, and $U_{\Sigma}, L_{\Sigma}$, denote the upper and lower estimates for the total volume of the inhomogenities in the case of one measurement $\left(\tilde{D}^{(1)}\right)$ and two measurements $\left(\tilde{D}^{(1)}+\tilde{D}^{(2)}\right)$ respectively. Figure 2 provides a 
comparison of the four estimates by displaying the "effectivities"

$$
\begin{aligned}
& (A): U_{1} /\left|\omega_{\epsilon}\right|=\max \left\{\gamma_{0}, \gamma_{1}\right\} \frac{\rho+1}{K} \sum_{j=1}^{K}\left[\frac{\cos ^{2} \theta_{j}}{\gamma_{0} \rho+\gamma_{1}}+\frac{\sin ^{2} \theta_{j}}{\gamma_{0}+\gamma_{1} \rho}\right], \\
& \text { (B) }: L_{1} /\left|\omega_{\epsilon}\right|=\min \left\{\gamma_{0}, \gamma_{1}\right\} \frac{\rho+1}{K} \sum_{j=1}^{K}\left[\frac{\cos ^{2} \theta_{j}}{\gamma_{0} \rho+\gamma_{1}}+\frac{\sin ^{2} \theta_{j}}{\gamma_{0}+\gamma_{1} \rho}\right],
\end{aligned}
$$

and

$$
\begin{aligned}
& (C): U_{\Sigma} /\left|\omega_{\epsilon}\right|=\frac{(1+\rho)^{2}\left(\gamma_{0}+\gamma_{1}\right)^{2}}{4\left(\gamma_{0} \rho+\gamma_{1}\right)\left(\gamma_{0}+\gamma_{1} \rho\right)}, \\
& (D): L_{\Sigma} /\left|\omega_{\epsilon}\right|=\frac{(1+\rho)^{2} \gamma_{0} \gamma_{1}}{\left(\gamma_{0} \rho+\gamma_{1}\right)\left(\gamma_{0}+\gamma_{1} \rho\right)},
\end{aligned}
$$

as functions of the ellipse aspect ratio, $\rho$. The closer the effectivity is to 1 the better the estimate - asymptotic lower bounds have effectivity $\leq 1$, asymptotic upper bounds effectivity $\geq 1$. The effectivities of the estimates using two measurements ((C) and (D)) are independent of the orientations of the ellipses. The effectivities of the estimates using one measurement depend on the set of rotations $\theta_{j}$ : for each value of the aspect ratio we have generated 100 random sets of rotations of the $K(=10)$ ellipses. Each point of "the cluster" around curve A represents the effectivity of a one-measurement upper bound each point of "the cluster" around curve B represents the effectivity of a one-measurement lower bound. The curves A and B are the graphs of

$$
\bar{U}_{1} /\left|\omega_{\epsilon}\right|=\max \left\{\gamma_{0}, \gamma_{1}\right\} \frac{(\rho+1)^{2}\left(\gamma_{0}+\gamma_{1}\right)}{2\left(\gamma_{0} \rho+\gamma_{1}\right)\left(\gamma_{0}+\gamma_{1} \rho\right)},
$$

and

$$
\bar{L}_{1} /\left|\omega_{\epsilon}\right|=\min \left\{\gamma_{0}, \gamma_{1}\right\} \frac{(\rho+1)^{2}\left(\gamma_{0}+\gamma_{1}\right)}{2\left(\gamma_{0} \rho+\gamma_{1}\right)\left(\gamma_{0}+\gamma_{1} \rho\right)} .
$$

These represent the average bounds (average effectivities) supposing the rotations are independent and uniformly distributed. These may also be obtained as an average of the bound using measurement $\tilde{D}^{(1)}$ and the bound using measurement $\tilde{D}^{(2)}$ for a fixed (but arbitrary) set of orientations. The fairest comparison of the effectivities using one and two measurements is probably to take the effectivity of the minimal upper bound obtained using $\tilde{D}^{(1)}$ and $\tilde{D}^{(2)}$, and the effectivity of the maximal lower bound obtained using $\tilde{D}^{(1)}$ and $\tilde{D}^{(2)}$, and compare these to the effectivities $U_{\Sigma} /\left|\omega_{\epsilon}\right|$ and $L_{\Sigma} /\left|\omega_{\epsilon}\right|$. In this context we note that points of "the top cluster" below the curve A and points of "the bottom cluster" above the curve B represent such minimal and maximal bounds, respectively. For the computation presented here we have taken $\gamma_{0}=1$ and $\gamma_{1}=3$. Figure 2 quite clearly documents the improvement in the estimates obtained using two simultaneous measurements, when compared to those using a single measurement.

The bounds in the estimate (6) for the trace of the polarization tensor $M$ are achieved for circular and "thin strip" inhomogeneities, respectively. In terms of the two-measurement volume estimates this implies that the upper estimate is asymptotically exact for circular inhomogeneities $(\rho=1)$ and that the lower estimate is asymptotically exact for "thin strip" inhomogeneities $(\rho \rightarrow \infty)$. Figure 2 clearly confirms this assertion.

Our last set of experiments provide a comparison of the accuracy of the four estimates in a fairly generic two dimensional setting. The sets of inhomogenities are finite unions of (in this case 10) ellipses. Figure 3 shows a typical configuration.

The data, $D^{(i)}, i=1,2$, which we need for the one-measurement and two-measurement volume estimates, are generated by (approximately) solving the corresponding elliptic boundary value problems (using a boundary 


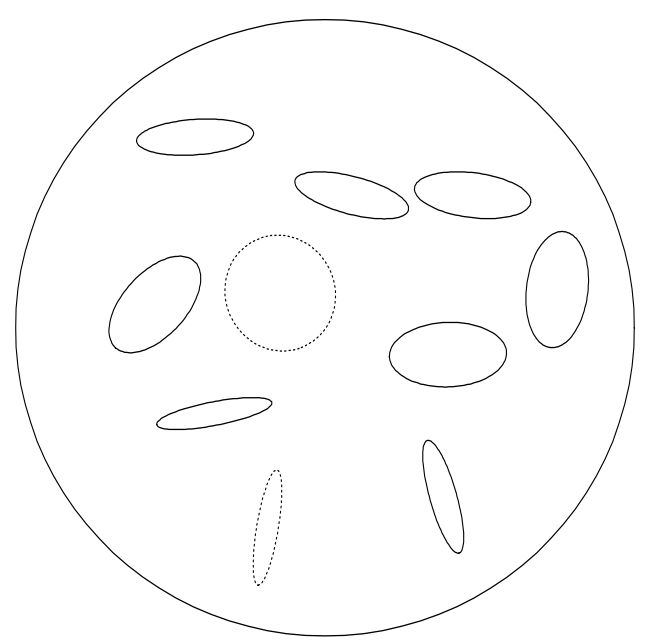

FiguRE 3. Typical configuration. The "dotted" curves indicate the two ellipses with the "most extreme" aspect ratios.
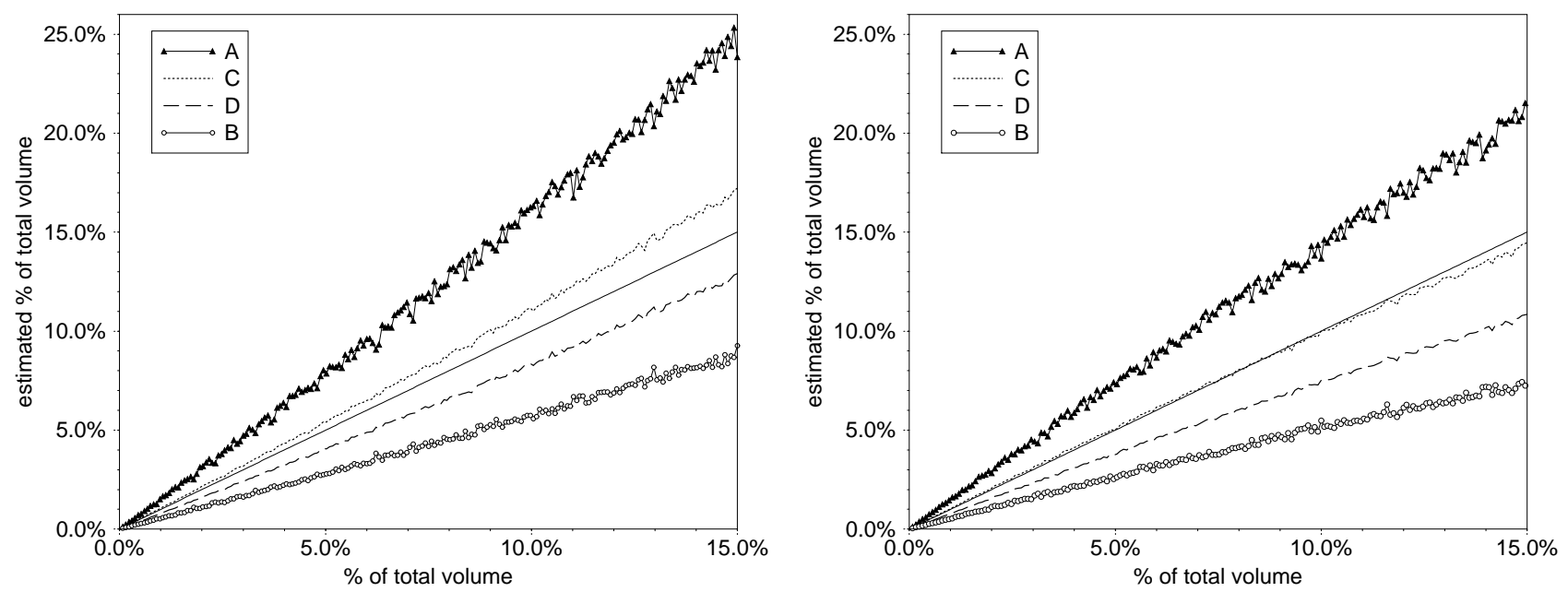

FIGURE 4. The four volume estimates in the case of 10 "random" elliptical inhomogeneities. The background conductivity, $\gamma_{0}$, is 1 . For the left graph $\gamma_{1}$, the conductivity of the inhomogeneities, is $1 / 3$, for the right graph $\gamma_{1}$ is 3 . The curves $\mathrm{A}$ and $\mathrm{B}$ represent the one-measurement estimates (8), the curves C and D the two-measurement estimates (9).

integral formulation). The volume of inhomogeneities ranges from very small to $15 \%$ of the volume of the full domain (the unit disk). Given a desired volume fraction we select the aspect ratios (between 1 and 6) the locations, and the orientations of the ellipses randomly. The (length of the) major axis of the (10) ellipses is then taken to be the same, the exact value determined from the desired volume fraction. Constraints are built in to assure that the ellipses are well separated and away from the boundary. In Figure 4 we display the curves corresponding to the four estimates (and the straight line corresponding to the exact value of $\left.\left|\omega_{\epsilon}\right|\right)$ for volume fractions between 0 and $15 \%$. In the case of the one-measurement estimates we display the best upper estimate, and the best lower estimate arising from the two data, $D^{(i)}, i=1,2$ (i.e. the minimum of the two upper estimates and the maximum of the two lower estimates, respectively). This figure clearly illustrates our assertion that the two-measurement estimates represent a significant improvement (over the one-measurement 
estimates) even for moderate size inhomogeneity volume fractions. We notice a certain similarity between the generic behaviour illustrated in Figure 4 and the behaviour observed for the rather special example in Figure 1 (as far as volume fractions below 15\% are concerned). The fact that it is (again) the upper estimate that fails to be a strict bound can be attributed to our aspect ratio restriction (between 1 and 6) which admits circles but not "very thin strips".

\section{BOUNDS FOR THE POLARIZATION TENSOR}

According to [8] the tensor valued field $M(x)$ in the representation formula (4) is defined by the (limiting) relationship

$$
\int_{\Omega} M_{i j} \xi_{i} \xi_{j} \phi \mathrm{d} \mu=\left|\omega_{\epsilon_{n}}\right|^{-1} \int_{\omega_{\epsilon_{n}}} \nabla v_{\epsilon_{n}} \cdot \nabla V \phi \mathrm{d} x+o(1)
$$

for any $\phi \in C^{0}(\bar{\Omega})$. Here $V$ and $v_{\epsilon}$ are solutions to

$$
\nabla \cdot\left(\gamma_{0} \nabla V\right)=\nabla \cdot\left(\gamma_{0} \xi\right) \quad \text { in } \Omega, \quad \gamma_{0} \frac{\partial V}{\partial n}=\gamma_{0} \xi \cdot n \quad \text { on } \partial \Omega
$$

and

$$
\nabla \cdot\left(\hat{\gamma}_{\epsilon} \nabla v_{\epsilon}\right)=\nabla \cdot\left(\gamma_{0} \xi\right) \quad \text { in } \Omega, \quad \hat{\gamma}_{\epsilon} \frac{\partial v_{\epsilon}}{\partial n}=\gamma_{0} \xi \cdot n \quad \text { on } \partial \Omega
$$

We note that $V$ has a very simple formula, namely $V(x)=\xi \cdot x+$ const. The probability measure $\mu$ is the limit (in the sense of measures) of $\frac{1}{\left|\omega_{\epsilon_{n}}\right|} 1_{\omega_{\epsilon_{n}}}$. Since the sets $\omega_{\epsilon}$ all lie inside a smooth compact set $K$, the support of the measure $\mu$ lies inside the same set $K \subset \subset \Omega$. The limiting relationship defining $M$ may thus be written

$$
\int_{K} M_{i j} \xi_{i} \xi_{j} \phi \mathrm{d} \mu=\left|\omega_{\epsilon_{n}}\right|^{-1} \int_{\omega_{\epsilon_{n}}} \nabla v_{\epsilon_{n}} \cdot \nabla V \phi \mathrm{d} x+o(1)
$$

for any $\phi \in C^{0}$. It is very useful to note that the tensor $M$ is completely independent of the domain $\Omega$.

Proposition 1. Suppose $\omega_{\epsilon} \subset \subset K \subset \subset \Omega_{1} \subset \Omega_{2}$, and let $v_{\epsilon}^{(i)}$ denote the solutions to (10) corresponding to the domains $\Omega_{i}, i=1,2$, for some fixed vector $\xi$. Then

$$
\left|\omega_{\epsilon}\right|^{-1} \int_{\omega_{\epsilon}} \nabla v_{\epsilon}^{(1)} \cdot \nabla V \phi \mathrm{d} x=\left|\omega_{\epsilon}\right|^{-1} \int_{\omega_{\epsilon}} \nabla v_{\epsilon}^{(2)} \cdot \nabla V \phi \mathrm{d} x+o(1)
$$

for any $\phi \in C^{0}$.

Proof of Proposition 1. The second estimate in Lemma 1 of [8] asserts that, given any $\eta>0$,

$$
\left\|v_{\epsilon}^{(2)}-V^{(2)}\right\|_{L^{2}\left(\Omega_{2}\right)} \leq C_{\eta}\left|\omega_{\epsilon}\right|^{\frac{1}{2}+\frac{1}{m *}-\eta}
$$

with $m *=\max \{m, 2\}$. Here $V^{(2)}(x)=\xi \cdot x+c_{2}$, with $c_{2}$ chosen so that $\int_{\partial \Omega_{2}} V^{(2)} \mathrm{d} \sigma=0$. Let $D=\left\{x \in \Omega_{2}\right.$ : $\left.\operatorname{dist}\left(x, \partial \Omega_{1}\right)<\delta\right\}$ be a sufficiently small neighborhood of $\partial \Omega_{1}$. Since the function $v_{\epsilon}^{(2)}-V^{(2)}$ is $\gamma_{0}$-harmonic outside $K$, elliptic estimates imply that

$$
\left\|v_{\epsilon}^{(2)}-V^{(2)}\right\|_{C^{1}(D)} \leq C_{\eta}\left|\omega_{\epsilon}\right|^{\frac{1}{2}+\frac{1}{m *}-\eta} .
$$


Hence

$$
\left\|\hat{\gamma}_{\epsilon} \frac{\partial v_{\epsilon}^{(2)}}{\partial n}-\hat{\gamma}_{\epsilon} \frac{\partial v_{\epsilon}^{(1)}}{\partial n}\right\|_{C^{0}\left(\partial \Omega_{1}\right)}=\left\|\gamma_{0} \frac{\partial v_{\epsilon}^{(2)}}{\partial n}-\gamma_{0} \frac{\partial V^{(2)}}{\partial n}\right\|_{C^{0}\left(\partial \Omega_{1}\right)} \leq C_{\eta}\left|\omega_{\epsilon}\right|^{\frac{1}{2}+\frac{1}{m *}-\eta}
$$

and therefore, by energy estimates

$$
\left\|\nabla v_{\epsilon}^{(2)}-\nabla v_{\epsilon}^{(1)}\right\|_{L^{2}\left(\Omega_{1}\right)} \leq C\left\|\hat{\gamma}_{\epsilon} \frac{\partial v_{\epsilon}^{(2)}}{\partial n}-\hat{\gamma}_{\epsilon} \frac{\partial v_{\epsilon}^{(1)}}{\partial n}\right\|_{H^{-1 / 2}\left(\partial \Omega_{1}\right)} \leq C_{\eta}\left|\omega_{\epsilon}\right|^{\frac{1}{2}+\frac{1}{m *}-\eta} .
$$

This last inequality immediately implies that

$$
\begin{aligned}
\left.|| \omega_{\epsilon}\right|^{-1} \int_{\omega_{\epsilon}}\left(\nabla v_{\epsilon}^{(2)}-\nabla v_{\epsilon}^{(1)}\right) \cdot \nabla V \phi \mathrm{d} x \mid & \leq C\left|\omega_{\epsilon}\right|^{-1}\left\|\nabla v_{\epsilon}^{(2)}-\nabla v_{\epsilon}^{(1)}\right\|_{L^{2}\left(\Omega_{1}\right)}\left|\omega_{\epsilon}\right|^{\frac{1}{2}} \\
& \leq C_{\eta}\left|\omega_{\epsilon}\right|^{\frac{1}{m *}-\eta},
\end{aligned}
$$

as desired.

We now suppose that $\gamma_{0}, \gamma_{1}$, and $M$ are constant (the latter naturally only on the support of $\mu$ ) with $\gamma_{0} \neq \gamma_{1}$. This assumption is conceptually not very restrictive, but technically it simplifies the proof of the bounds (6) for the trace of $M$, since it eliminates any need for localization. By inserting $\phi=\gamma_{1}-\gamma_{0}$ in the identity (11) we now obtain

$$
\left(\gamma_{1}-\gamma_{0}\right) M_{i j} \xi_{i} \xi_{j}=\left|\omega_{\epsilon_{n}}\right|^{-1} \int_{\omega_{\epsilon_{n}}}\left(\gamma_{1}-\gamma_{0}\right) \nabla v_{\epsilon_{n}} \cdot \nabla V \mathrm{~d} x+o(1)
$$

We may rewrite

$$
\begin{aligned}
\left|\omega_{\epsilon}\right|^{-1} \int_{\omega_{\epsilon}}\left(\gamma_{1}-\gamma_{0}\right) \nabla v_{\epsilon} \cdot \nabla V \mathrm{~d} x & =\left|\omega_{\epsilon}\right|^{-1} \int_{\omega_{\epsilon}}\left(\gamma_{1}-\gamma_{0}\right)|\nabla V|^{2} \mathrm{~d} x+\left|\omega_{\epsilon}\right|^{-1} \int_{\omega_{\epsilon}}\left(\gamma_{1}-\gamma_{0}\right)\left(\nabla v_{\epsilon}-\nabla V\right) \cdot \nabla V \mathrm{~d} x \\
& =\left|\omega_{\epsilon}\right|^{-1} \int_{\omega_{\epsilon}}\left(\gamma_{1}-\gamma_{0}\right)|\nabla V|^{2} \mathrm{~d} x+\left|\omega_{\epsilon}\right|^{-1} \int_{\omega_{\epsilon}}\left(\gamma_{1}-\gamma_{0}\right) \nabla w_{\epsilon} \cdot \nabla V \mathrm{~d} x,
\end{aligned}
$$

where $w_{\epsilon}=v_{\epsilon}-V$ is the solution to

$$
\nabla \cdot\left(\hat{\gamma}_{\epsilon} \nabla w_{\epsilon}\right)=\nabla \cdot\left(\left(\gamma_{0}-\gamma_{1}\right) 1_{\omega_{\epsilon}} \xi\right) \quad \text { in } \Omega, \quad \hat{\gamma}_{\epsilon} \frac{\partial w_{\epsilon}}{\partial n}=0 \quad \text { on } \partial \Omega .
$$

Alternatively $w_{\epsilon}$ is the minimizer (in $H^{1}(\Omega)$ ) of the energy expression

$$
\int_{\Omega} \hat{\gamma}_{\epsilon}\left|\nabla w+\frac{\left(\gamma_{1}-\gamma_{0}\right)}{\gamma_{1}} 1_{\omega_{\epsilon}} \xi\right|^{2} \mathrm{~d} x
$$

and

$$
\begin{aligned}
\min _{w \in H^{1}(\Omega)} \int_{\Omega} \hat{\gamma}_{\epsilon}\left|\nabla w+\frac{\left(\gamma_{1}-\gamma_{0}\right)}{\gamma_{1}} 1_{\omega_{\epsilon}} \xi\right|^{2} \mathrm{~d} x & =\int_{\Omega} \hat{\gamma}_{\epsilon}\left|\nabla w_{\epsilon}+\frac{\left(\gamma_{1}-\gamma_{0}\right)}{\gamma_{1}} 1_{\omega_{\epsilon}} \xi\right|^{2} \mathrm{~d} x \\
& =\int_{\omega_{\epsilon}} \frac{\left(\gamma_{1}-\gamma_{0}\right)^{2}}{\gamma_{1}}|\xi|^{2} \mathrm{~d} x+\int_{\omega_{\epsilon}}\left(\gamma_{1}-\gamma_{0}\right) \nabla w_{\epsilon} \cdot \xi \mathrm{d} x
\end{aligned}
$$


so that

$$
\begin{aligned}
\left|\omega_{\epsilon}\right|^{-1} \int_{\omega_{\epsilon}}\left(\gamma_{1}-\gamma_{0}\right) \nabla v_{\epsilon} \cdot \nabla V \mathrm{~d} x & =\left|\omega_{\epsilon}\right|^{-1} \int_{\omega_{\epsilon}}\left(\gamma_{1}-\gamma_{0}\right)|\nabla V|^{2} \mathrm{~d} x+\left|\omega_{\epsilon}\right|^{-1} \int_{\omega_{\epsilon}}\left(\gamma_{1}-\gamma_{0}\right) \nabla w_{\epsilon} \cdot \nabla V \mathrm{~d} x \\
& =\left(\gamma_{1}-\gamma_{0}\right) \frac{\gamma_{0}}{\gamma_{1}}|\xi|^{2}+\left|\omega_{\epsilon}\right|^{-1} \min _{w \in H^{1}(\Omega)} \int_{\Omega} \hat{\gamma}_{\epsilon}\left|\nabla w+\frac{\left(\gamma_{1}-\gamma_{0}\right)}{\gamma_{1}} 1_{\omega_{\epsilon}} \xi\right|^{2} \mathrm{~d} x
\end{aligned}
$$

In other words we have

$$
\left(\gamma_{1}-\gamma_{0}\right) M_{i j} \xi_{i} \xi_{j}=\left(\gamma_{1}-\gamma_{0}\right) \frac{\gamma_{0}}{\gamma_{1}}|\xi|^{2}+\left|\omega_{\epsilon_{n}}\right|^{-1} \min _{w \in H^{1}(\Omega)} \int_{\Omega} \hat{\gamma}_{\epsilon_{n}}\left|\nabla w+\frac{\left(\gamma_{1}-\gamma_{0}\right)}{\gamma_{1}} 1_{\omega_{\epsilon_{n}}} \xi\right|^{2} \mathrm{~d} x+o(1) .
$$

From this identity we immediately conclude that

$$
\left(\gamma_{1}-\gamma_{0}\right) \frac{\gamma_{0}}{\gamma_{1}}|\xi|^{2} \leq\left(\gamma_{1}-\gamma_{0}\right) M_{i j} \xi_{i} \xi_{j} \leq\left(\gamma_{1}-\gamma_{0}\right)|\xi|^{2}
$$

or

$$
\min \left\{1, \frac{\gamma_{0}}{\gamma_{1}}\right\} I \leq M \leq \max \left\{1, \frac{\gamma_{0}}{\gamma_{1}}\right\} I
$$

as was proven (in its stronger, local form) in [8]. As we have already seen these bounds for the polarization tensor $M$ immediately lead to optimal asymptotic bounds for $\left|\omega_{\epsilon}\right|$ in terms of a single boundary integral. When it comes to establishing optimal asymptotic bounds for $\left|\omega_{\epsilon}\right|$ in terms of multiple boundary integrals (as we did earlier in this paper) then we need optimal, geometry independent bounds for the trace of $M$. In this connection we note that the trace bounds $m \min \left\{1, \frac{\gamma_{0}}{\gamma_{1}}\right\}$ and $m \max \left\{1, \frac{\gamma_{0}}{\gamma_{1}}\right\}$ which follow directly from (13) are not optimal. Indeed, the optimal bounds are

$$
\frac{m^{2} \gamma_{0}}{\gamma_{1}+(m-1) \gamma_{0}}=m h\left(\frac{\gamma_{0}}{\gamma_{1}}\right) \leq \operatorname{trace}(M) \leq m a\left(\frac{\gamma_{0}}{\gamma_{1}}\right)=\frac{(m-1) \gamma_{1}+\gamma_{0}}{\gamma_{1}}
$$

The remainder of this section is devoted to a derivation of (14). We have already observed that the tensor $M$ is independent of the domain $\Omega$; due to the variational characterization (12) this asserts that the value

$$
\left|\omega_{\epsilon}\right|^{-1} \min _{w \in H^{1}(\Omega)} \int_{\Omega} \hat{\gamma}_{\epsilon}\left|\nabla w+\frac{\left(\gamma_{1}-\gamma_{0}\right)}{\gamma_{1}} 1_{\omega_{\epsilon}} \xi\right|^{2} \mathrm{~d} x
$$

is independent of $\Omega$, modulo terms of order $o(1)$. In the same fashion we can prove that the above minimum (modulo terms of order $o(1)$ ) is "independent of the boundary conditions" placed on $w$, in the sense that we may replace $H^{1}(\Omega)$ by $H_{0}^{1}(\Omega)=\left\{w \in H^{1}(\Omega): w=0\right.$ on $\left.\partial \Omega\right\}$ or $H_{\text {per }}^{1}=\left\{w \in H_{\text {loc }}^{1}: w\left(x+T e_{j}\right)=w(x), j=1 \ldots m\right\}$ (the latter when $\Omega$ is the taken to be a sufficiently large cube $\Omega=Q=[0, T]^{m}$ ). We shall use the ensuing characterization

$$
\left(\gamma_{1}-\gamma_{0}\right) M_{i j} \xi_{i} \xi_{j}=\left(\gamma_{1}-\gamma_{0}\right) \frac{\gamma_{0}}{\gamma_{1}}|\xi|^{2}+\left|\omega_{\epsilon_{n}}\right|^{-1} \min _{w \in H_{\mathrm{per}}^{1}(Q)} \int_{Q} \hat{\gamma}_{\epsilon_{n}}\left|\nabla w+\frac{\left(\gamma_{1}-\gamma_{0}\right)}{\gamma_{1}} 1_{\omega_{\epsilon}} \xi\right|^{2} \mathrm{~d} x+o(1)
$$

together with the Hashin-Shtrikman variational technique described by Kohn and Milton (cf. [14]) to derive the improved bounds (14) for the trace of the tensor $M$. For completeness and the convenience of the reader we provide some of the details. 
Sketch of proof of (14). By introducing a constant reference medium (with conductivity $c<\min \left\{\gamma_{0}, \gamma_{1}\right\}$ ), by writing the part of the energy which pertains to the "positive" conductivity perturbation $\hat{\gamma}_{\epsilon}-c$ as a maximum over "dual fields", and by interchanging the order of the minimization and the maximization (which can be justified) we arrive at the following variational principle

$$
\begin{aligned}
\min _{w \in H_{\mathrm{per}}^{1}(Q)} \int_{Q} \hat{\gamma}_{\epsilon}\left|\nabla w+\frac{\left(\gamma_{1}-\gamma_{0}\right)}{\gamma_{1}} 1_{\omega_{\epsilon}} \xi\right|^{2} \mathrm{~d} x= & c\left|\omega_{\epsilon}\right| \frac{\left(\gamma_{1}-\gamma_{0}\right)^{2}}{\gamma_{1}^{2}}|\xi|^{2}+\sup _{\sigma \in L_{\mathrm{per}}^{2}}\left[-\int_{Q}\left(\hat{\gamma}_{\epsilon}-c\right)^{-1}|\sigma|^{2} \mathrm{~d} x\right. \\
& +2 \int_{Q} \frac{\left(\gamma_{1}-\gamma_{0}\right)}{\gamma_{1}} 1_{\omega_{\epsilon}} \xi \cdot \sigma \mathrm{d} x \\
& \left.+c \int_{Q}\left\langle L\left(\frac{1}{c} \sigma+\frac{\left(\gamma_{1}-\gamma_{0}\right)}{\gamma_{1}} 1_{\omega_{\epsilon}} \xi\right), \frac{1}{c} \sigma+\frac{\left(\gamma_{1}-\gamma_{0}\right)}{\gamma_{1}} 1_{\omega_{\epsilon}} \xi\right\rangle \mathrm{d} x\right],
\end{aligned}
$$

where $L$ denotes the operator $L=-\nabla \Delta^{-1} \nabla \cdot, \Delta^{-1}$, being the inverse of the Laplace operator with periodic boundary conditions. Similarly, by introducing a constant reference medium (with conductivity $c>$ $\left.\max \left\{\gamma_{0}, \gamma_{1}\right\}\right)$, by writing the part of the energy which pertains to the "negative" conductivity perturbation $\hat{\gamma}_{\epsilon}-c$ as a minimum over "dual fields", and by interchanging the order of the two minimizations, we arrive at the variational principle

$$
\begin{aligned}
\min _{w \in H_{\mathrm{per}}^{1}(Q)} \int_{Q} \hat{\gamma}_{\epsilon}\left|\nabla w+\frac{\left(\gamma_{1}-\gamma_{0}\right)}{\gamma_{1}} 1_{\omega_{\epsilon}} \xi\right|^{2} \mathrm{~d} x= & c\left|\omega_{\epsilon}\right| \frac{\left(\gamma_{1}-\gamma_{0}\right)^{2}}{\gamma_{1}^{2}}|\xi|^{2}+\inf _{\sigma \in L_{\mathrm{per}}^{2}}\left[-\int_{Q}\left(\hat{\gamma}_{\epsilon}-c\right)^{-1}|\sigma|^{2} \mathrm{~d} x\right. \\
& +2 \int_{Q} \frac{\left(\gamma_{1}-\gamma_{0}\right)}{\gamma_{1}} 1_{\omega_{\epsilon}} \xi \cdot \sigma \mathrm{d} x \\
& \left.+c \int_{Q}\left\langle L\left(\frac{1}{c} \sigma+\frac{\left(\gamma_{1}-\gamma_{0}\right)}{\gamma_{1}} 1_{\omega_{\epsilon}} \xi\right), \frac{1}{c} \sigma+\frac{\left(\gamma_{1}-\gamma_{0}\right)}{\gamma_{1}} 1_{\omega_{\epsilon}} \xi\right\rangle \mathrm{d} x\right] .
\end{aligned}
$$

If we now use only test functions of the form $\sigma=\lambda 1_{\omega_{\epsilon}} \xi$ in these two variational principles (as opposed to arbitrary $\sigma \in L_{\text {per }}^{2}$ ) then we arrive at the inequalities

$$
\begin{array}{r}
\min _{w \in H_{\mathrm{per}}^{1}(Q)} \int_{\Omega} \hat{\gamma}_{\epsilon}\left|\nabla w+\frac{\left(\gamma_{1}-\gamma_{0}\right)}{\gamma_{1}} 1_{\omega_{\epsilon}} \xi\right|^{2} \mathrm{~d} x \geq c\left|\omega_{\epsilon}\right| \frac{\left(\gamma_{1}-\gamma_{0}\right)^{2}}{\gamma_{1}^{2}}|\xi|^{2}+\max _{\lambda \in \mathbf{R}}\left[-\left|\omega_{\epsilon}\right|\left(\gamma_{1}-c\right)^{-1} \lambda^{2}|\xi|^{2}\right. \\
\left.+2\left|\omega_{\epsilon}\right| \frac{\left(\gamma_{1}-\gamma_{0}\right)}{\gamma_{1}} \lambda|\xi|^{2}+\frac{1}{c}\left(\lambda+c \frac{\left(\gamma_{1}-\gamma_{0}\right)}{\gamma_{1}}\right)^{2}\left\langle A_{\epsilon} \xi, \xi\right\rangle\right]
\end{array}
$$

for $c<\min \left\{\gamma_{0}, \gamma_{1}\right\}$, and

$$
\begin{aligned}
\min _{w \in H_{\mathrm{per}}^{1}(Q)} \int_{\Omega} \hat{\gamma}_{\epsilon}\left|\nabla w+\frac{\left(\gamma_{1}-\gamma_{0}\right)}{\gamma_{1}} 1_{\omega_{\epsilon}} \xi\right|^{2} \mathrm{~d} x \leq & c\left|\omega_{\epsilon}\right| \frac{\left(\gamma_{1}-\gamma_{0}\right)^{2}}{\gamma_{1}^{2}}|\xi|^{2}+\min _{\lambda \in \mathbf{R}}\left[-\left|\omega_{\epsilon}\right|\left(\gamma_{1}-c\right)^{-1} \lambda^{2}|\xi|^{2}\right. \\
& \left.+2\left|\omega_{\epsilon}\right| \frac{\left(\gamma_{1}-\gamma_{0}\right)}{\gamma_{1}} \lambda|\xi|^{2}+\frac{1}{c}\left(\lambda+c \frac{\left(\gamma_{1}-\gamma_{0}\right)}{\gamma_{1}}\right)^{2}\left\langle A_{\epsilon} \xi, \xi\right\rangle\right]
\end{aligned}
$$

for $c>\max \left\{\gamma_{0}, \gamma_{1}\right\}$, respectively. In both cases $A_{\epsilon}$ denotes the symmetric matrix given by $\left(A_{\epsilon}\right)_{i j}=$ $-\int_{Q} \frac{\partial}{\partial x_{j}} \Delta^{-1}\left(\frac{\partial}{\partial x_{i}} 1_{\omega_{\epsilon}}\right) 1_{\omega_{\epsilon}} \mathrm{d} x$. Due to the fact that we impose periodic boundary conditions (as opposed to 
Dirichlet or Neumann boundary conditions) we have that $\Delta^{-1}\left(\frac{\partial}{\partial x_{i}} 1_{\omega_{\epsilon}}\right)=\frac{\partial}{\partial x_{i}} \Delta^{-1}\left(1_{\omega_{\epsilon}}-\frac{\left|\omega_{\epsilon}\right|}{|Q|}\right)$, and therefore

$$
\operatorname{trace}\left(A_{\epsilon}\right)=-\int_{Q}\left(1_{\omega_{\epsilon}}-\frac{\left|\omega_{\epsilon}\right|}{|Q|}\right) 1_{\omega_{\epsilon}} \mathrm{d} x=-\left|\omega_{\epsilon}\right|+O\left(\left|\omega_{\epsilon}\right|^{2}\right)
$$

In combination with (15) the estimate (16) now yields

$$
\begin{aligned}
\left(\gamma_{1}-\gamma_{0}\right) \operatorname{trace}(M) \geq & m\left(\gamma_{1}-\gamma_{0}\right) \frac{\gamma_{0}}{\gamma_{1}}+m c \frac{\left(\gamma_{1}-\gamma_{0}\right)^{2}}{\gamma_{1}^{2}} \\
& +\max _{\lambda \in \mathbf{R}}\left[-m\left(\gamma_{1}-c\right)^{-1} \lambda^{2}+2 m \frac{\left(\gamma_{1}-\gamma_{0}\right)}{\gamma_{1}} \lambda-\frac{1}{c}\left(\lambda+c \frac{\left(\gamma_{1}-\gamma_{0}\right)}{\gamma_{1}}\right)^{2}\right] \\
= & \frac{\left(\gamma_{1}-\gamma_{0}\right)}{\gamma_{1}}\left(m \gamma_{0}+(m-1) c \frac{\left(\gamma_{1}-\gamma_{0}\right)}{\gamma_{1}}+\frac{(m-1)^{2}\left(\gamma_{1}-\gamma_{0}\right)\left(\gamma_{1}-c\right) c}{\left((m-1) c+\gamma_{1}\right) \gamma_{1}}\right)
\end{aligned}
$$

for any $c<\min \left\{\gamma_{0}, \gamma_{1}\right\}$. Similarly a combination of (15) and the estimate (17) yields

$$
\begin{aligned}
\left(\gamma_{1}-\gamma_{0}\right) \operatorname{trace}(M) \leq & m\left(\gamma_{1}-\gamma_{0}\right) \frac{\gamma_{0}}{\gamma_{1}}+m c \frac{\left(\gamma_{1}-\gamma_{0}\right)^{2}}{\gamma_{1}^{2}} \\
& +\min _{\lambda \in \mathbf{R}}\left[-m\left(\gamma_{1}-c\right)^{-1} \lambda^{2}+2 m \frac{\left(\gamma_{1}-\gamma_{0}\right)}{\gamma_{1}} \lambda-\frac{1}{c}\left(\lambda+c \frac{\left(\gamma_{1}-\gamma_{0}\right)}{\gamma_{1}}\right)^{2}\right] \\
= & \frac{\left(\gamma_{1}-\gamma_{0}\right)}{\gamma_{1}}\left(m \gamma_{0}+(m-1) c \frac{\left(\gamma_{1}-\gamma_{0}\right)}{\gamma_{1}}+\frac{(m-1)^{2}\left(\gamma_{1}-\gamma_{0}\right)\left(\gamma_{1}-c\right) c}{\left((m-1) c+\gamma_{1}\right) \gamma_{1}}\right)
\end{aligned}
$$

for any $c>\max \left\{\gamma_{0}, \gamma_{1}\right\}$. Since the left hand side, $\left(\gamma_{1}-\gamma_{0}\right)$ trace $(M)$, in each of these two bounds is independent of $c$, the limiting bounds corresponding to $c=\min \left\{\gamma_{0}, \gamma_{1}\right\}$, and $c=\max \left\{\gamma_{0}, \gamma_{1}\right\}$ respectively, are also valid. Very simple manipulations show that these limiting bounds are equivalent to (14).

Remark 1. A very related, but slightly different approach to derive the bounds (14) is to associate the polarization tensor with the first order term in a low volume fraction perturbation of the effective tensor of a two component mixture (with condutivities $\gamma_{0}$ and $\gamma_{1}$ ) and then perform an expansion (to first order) of the classical Hashin-Shtrikman bounds, as derived for instance in [14]. This second approach has been carried out in [16], resulting in geometry dependent bounds for the entire tensor $M$. The (geometry independent) trace bounds (14) follow by fairly simple manipulations from the bounds derived in [16]. Concerning the optimality of the bounds (14), it is very easy to see that the lower bound is achieved for an inhomogeneity in the shape of a ball, in which case $M$ is given by $M=\frac{m \gamma_{0}}{\gamma_{1}+(m-1) \gamma_{0}} I$. One gets arbitrarily close to the upper bound by taking inhomogeneities in the shape of a thin sheets with thickness approaching 0 , see [5]; one may also use ellipsoids where one axis approaches 0 and the others stay fixed. For an explicit formula for $M$ in the case of an ellipsoid see [7] (for $m=2$ ) and [16] (for $m=3$ ).

Remark 2. In the absence of the assumption that $M$ be constant on the support of $\mu$ (but still assuming that $\gamma_{0}$ and $\gamma_{1}$ are different and constant) what we have really rigorously established is that $\int_{\Omega} \operatorname{trace}(M) \mathrm{d} \mu=$ $\int_{K} \operatorname{trace}(M) \mathrm{d} \mu$ satisfies

$$
m h\left(\frac{\gamma_{0}}{\gamma_{1}}\right) \leq \int_{\Omega} \operatorname{trace}(M) \mathrm{d} \mu \leq m a\left(\frac{\gamma_{0}}{\gamma_{1}}\right)
$$

For our application to $m$-mesurement estimates in this paper, these are really the only bounds we need, as noted near the end of Section 1. 
Acknowledgements. This research was partially supported by NSF grants DMS-0072556 and INT-0003788.

\section{REFERENCES}

[1] G. Alessandrini, E. Rosset and J.K. Seo, Optimal size estimates for the inverse conductivity problem with one measurement. Proc. Amer. Math. Soc. 128 (2000) 53-64.

[2] G. Alessandrini, A. Morassi and E. Rosset, Detecting cavities by electrostatic boundary measurements. Preprint (2002).

[3] H. Ammari and J.K. Seo, A new formula for the reconstruction of conductivity inhomogeneities. Preprint (2002).

[4] H. Ammari, S. Moskow and M.S. Vogelius, Boundary integral formulae for the reconstruction of electric and electromagnetic inhomogeneities of small volume. ESAIM: Cont. Opt. Calc. Var. 9 (2003) 49-66.

[5] E. Beretta, E. Francini and M.S. Vogelius, Asymptotic formulas for steady state voltage potentials in the presence of thin inhomogeneities. A rigorous error analysis. Preprint (2002).

[6] M. Brühl and M. Hanke, Numerical implementation of two noniterative methods for locating inclusions by impedance tomography. Inverse Problems 16 (2000) 1029-1042.

[7] M. Brühl, M. Hanke and M.S. Vogelius, A direct impedance tomography algorithm for locating small inhomogeneities. Numer. Math. 93 (2003) 635-654.

[8] Y. Capdeboscq and M.S. Vogelius, A general representation formula for boundary voltage perturbations caused by internal conductivity inhomogeneities of low volume fraction. ESAIM: M2AN 37 (2003) 159-173.

[9] D.J. Cedio-Fengya, S. Moskow and M.S. Vogelius, Identification of conductivity imperfections of small diameter by boundary measurements. Continuous dependence and computational reconstruction. Inverse Problems 14 (1998) 553-595.

[10] A. Friedman and V. Isakov, On the uniqueness in the inverse conductivity problem with one measurement. Indiana Univ. Math. J. 38 (1989) 553-580.

[11] S. He and V.G. Romanov, Identification of small flaws in conductors using magnetostatic measurements. Math. Comput. Simulation 50 (1999) 457-471.

[12] M. Ikehata and T. Ohe, A numerical method for finding the convex hull of polygonal cavities using the enclosure method. Inverse Problems 18 (2002) 111-124.

[13] H. Kang, J.K. Seo and D. Sheen, The inverse conductivity problem with one measurement: stability and estimation of size. SIAM J. Math. Anal. 28 (1997) 1389-1405.

[14] R.V. Kohn and G.W. Milton, On bounding the effective conductivity of anisotropic composites, in Homogenization and Effective Moduli of Materials and Media, J.L. Ericksen, D. Kinderlehrer, R. Kohn and J.-L. Lions Eds., Springer-Verlag, IMA Vol. Math. Appl. 1 (1986) 97-125.

[15] O. Kwon, J.K. Seo and J.-R. Yoon, A real time algorithm for the location search of discontinuous conductivities with one measurement. Comm. Pure Appl. Math. 55 (2002) 1-29.

[16] R. Lipton, Inequalities for electric and elastic polarization tensors with applications to random composites. J. Mech. Phys. Solids 41 (1993) 809-833.

To access this journal online:

www.edpsciences.org 